\title{
Gestión Pedagógica: Caso de una Universidad Pública
}

\author{
Fátima del Rosario Sánchez Pérez, \\ Dra. Minerva Camacho Javier,
} Universidad Juárez Autónoma de Tabasco, División Académica Ciencias Económico Administrativas, México

Doi:10.19044/esj.2020.v16n25p15 ～URL:http://dx.doi.org/10.19044/esj.2020.v16n25p15

\section{Resumen}

La presente investigación tiene como objeto de interés el análisis teórico-práctico de la gestión pedagógica desarrollada en una universidad pública ubicada al Sur de México. Abordada desde una visión cualitativa, la investigación documental se centró en una revisión específica sobre autores latinoamericanos que han investigado sobre la gestión educativa y pedagógica. Posteriormente, se contrastó la información documental con un proyecto de investigación longitudinal desarrollado por un grupo de docentes en una universidad pública ubicada al sur de México. Los resultados de este proyecto permitieron observar las fortalezas y las debilidades de desarrollo de la gestión pedagógica, denominado como un proceso profesionalizante en el fortalecimiento de una enseñanza-aprendizaje por competencias. Se requiere dar seguimiento a proyectos abocados a la gestión pedagógica a nivel regional o local, buscando que las políticas educativas en el nivel profesional puedan ser más certeras y congruentes ante la diferencia de realidades que se vive a lo largo de todo el país.

Palabras claves: Gestión educativa, Gestión pedagógica, Educación Superior, Calidad educativa 


\title{
Pedagogical Management: Case of a Public University
}

\author{
Fátima del Rosario Sánchez Pérez, \\ Dra. Minerva Camacho Javier, \\ Universidad Juárez Autónoma de Tabasco, \\ División Académica Ciencias Económico Administrativas, México
}

\begin{abstract}
The present research has as an object of interest the theoreticalpractical analysis of the pedagogical management developed in a public university located in the South of Mexico. Approached from a qualitative perspective, the documentary research focused on a specific review on Latin American authors who have investigated educational and pedagogical management. Subsequently, the documentary information was contrasted with a longitudinal research project developed by a group of teachers at a public university located in southern Mexico. The results of this project allowed to observe the strengths and weaknesses of pedagogical management development, called as a professionalizing process in the strengthening of teaching-learning by competencies. It is necessary to follow up on projects aimed at pedagogical management at the regional or local level, seeking that educational policies at the professional level can be more accurate and congruent in the face of the difference in realities that exist throughout the country.
\end{abstract}

Keywords: Education Management, Pedagogical Management, Higher Education, Educational Quality

\section{Introduction}

¿Qué tan preparados se encuentran los docentes de nivel superior en México para hacer frente a los diversos cambios que sufre en la actualidad el entorno educativo? ¿Qué competencias requiere fortalecer el docente de nivel superior? Estas son algunas de las preguntas que surgen ante la preocupación que manifiestan las Instituciones de Educación Superior (IES) en México, puesto que estas se ven inmersas en el desarrollo de estrategias para liderar la formación de profesionistas a la par de las tendencias globales; es decir, si no se cuenta con procesos administrativos y educativos integrales, estas tienden a desaparecer o pierden credibilidad educativa ante un mercado altamente competitivo y demandante. 
De esta manera, se determinó profundizar en la gestión pedagógica, con la idea de aportar soluciones a problemáticas educativas de carácter regional o local (UNESCO, 2017).

El interés es centrado en el concepto de gestión pedagógica ya que es la esencia para lograr innovación y dar cumplimiento a la misión educativa de las IES (Ibarra, 2016; Rodríguez, 2005). De la misma manera, esta investigación buscó entrelazar la investigación teórica con un proyecto desarrollado en una universidad pública de nivel superior ubicada al Sur de México. Se observó la intervención y aplicación de un proceso profesionalizante $^{1}$ en el desarrollo de las competencias docentes y el aprendizaje por competencias.

Por lo que los propósitos de esta investigación fueron: 1) Indagar sobre la gestión pedagógica, y la gestión educativa, definiendo y compilando un marco referencial abocado a trabajos realizados en Latinoamérica; 2) Analizar la gestión pedagógica dentro de un proceso profesionalizante desarrollado durante un estudio longitudinal en una universidad pública de nivel superior ubicada al Sur del país.

\section{Metodología}

El presente trabajo es de corte cualitativo de tipo exploratoriodescriptivo (Hernández, et al., 2014).

Respecto al tipo de estudio seleccionado, Behar (2008) explica que los estudios exploratorios son el comienzo para investigaciones específicas, se aplican cuando no se cuenta con suficientes antecedentes teóricos o prácticos y pueden complementarse con estudios descriptivos, estos últimos mediante un análisis complejo describen las características destacables de un evento, proceso o sujeto (Cortés \& Iglesias, 2004).

De esta manera, se buscó dar cumplimiento al propósito perseguido en esta investigación al entretejer la realidad a través del análisis de diversas fuentes de información teórica y un contexto educativo bajo un entorno mixto en vía de comprender cómo mejorar los procesos para alcanzar la calidad educativa por competencias.

\section{Procedimiento}

\section{Indagación documental}

El método utilizado fue el análisis documental, se delimitó el estudio mediante una clasificación de los trabajos elaborados sólo por autores latinoamericanos que han investigado la gestión educativa y la gestión

${ }^{1}$ Término esquematizado y aplicado por los investigadores a cargo del proyecto, el cual se encuentra certificado por el Registro Público del Derecho de Autor: 03-2019-01251224360001. Este proyecto continua vigente en la actualidad. 
pedagógica en los últimos cinco años. Lo anterior, conformó el denominado estado del arte.

Se optó por analizar los trabajos de autores latinoamericanos, ya que se considera la importancia que la UNESCO (2017) otorga a los estudios regionales: "Aunque pudiera resultar ser recomendable disponer de un marco mundial para el examen de los sistemas educativos y las políticas de educación, adoptar un enfoque regional o subregional es más factible" ( $p$. 422).

Se consultaron bases de datos oficiales como ResearchGate, Dialnet, Redalyc, SciELO y BASE, por el prestigio y confiabilidad de las publicaciones aceptadas. Otro aspecto tomado en cuenta respecto a las bases de datos seleccionadas es la tendencia de investigación de la mayoría de los trabajos, pues se enfocan a investigaciones aplicadas en Latinoamérica.

La búsqueda de las fuentes sobre gestión educativa, tuvo como principal criterio comprender los cambios y las modificaciones en la forma de administrar las IES, y la importancia de la gestión pedagógica dentro del proceso, ya que este elemento es el engrane principal para alcanzar la calidad en la educación.

Para facilitar la comprensión de la información, se diseñó un cuadro comparativo y se clasificó mediante el lugar de origen (internacional, nacional, local), el año y el tipo de documento (artículo, tesis, informe).

Mientras que, para la elaboración del marco teórico, fue utilizada la técnica de mapeo (Hernández, Fernández \& Baptista, 2014). Esta técnica consiste en realizar un mapa conceptual identificando y organizando los conceptos principales que abonan a la relevancia teórica de la investigación, la finalidad de esta técnica es delimitar y ordenar el contenido del trabajo.

\section{Indagación de campo}

Durante el ejercicio del servicio social, la investigación se abocó a observar a un grupo de docentes seleccionado mediante la técnica de muestreo no probabilístico denominada 'por conveniencia', debido al interés y accesibilidad de los sujetos en estudio (Otzen \& Manterola, 2017). Este grupo de docentes seleccionados formaron parte de un proyecto de investigaciónacción dentro de un entorno de participación y colaboración educativa mixto. El grupo de investigadores y docentes involucrados imparten asignaturas en el área Económico-Administrativas en una universidad pública del estado de Tabasco, su trabajo se enfoca en el desarrollo de las competencias docentes en un entorno mixto donde se observa el diseño y aplicación de la gestión pedagógica dentro de un proceso profesionalizante.

Se llevó un registro anecdótico sobre las reuniones de seguimiento y autoevaluación con los líderes del proyecto y los docentes participantes, esta técnica de observación directa es utilizada principalmente en el ámbito 
educativo para evaluar el aprendizaje de los alumnos, sin embargo también puede ser aplicado para registrar situaciones dentro de las instituciones educativas, consistiendo en un informe en el que se anota de manera objetiva los hechos observados de un grupo en específico con la finalidad de obtener 'datos útiles' para evaluar una determinada situación (Pardo et al., 2013).

Es importante mencionar que el objetivo de las reuniones del grupo de docentes participantes, contempladas dentro del proceso profesionalizante en el desarrollo de las competencias, fue para autoevaluar, reflexionar, retroalimentar, guiar e innovar en el proceso didáctico-pedagógico, respetando lo establecido en el modelo educativo de su universidad. Esta participación y colaboración entre los docentes, permitió un trabajo interdisciplinario importante medular para acercarse a una transformación y/o mejora de la calidad educativa.

\section{Resultados}

\section{Gestión Educativa (GE) y Gestión Pedagógica (GP)}

Resulta fundamental para esta investigación ahondar en los conceptos relevantes como lo son la GE y la GP, por lo que a continuación se presentan un cúmulo de definiciones que resultan ser concisas y afines a este trabajo.

La GE es considerada por algunos autores como una disciplina, tal es el caso de Casassus (2000) quien la define como: "una disciplina es gestación en la cual interactúan los planos de la teoría, los de la política y los de la pragmática" (p.2). Sin embargo, la mayoría de autores estudiados convergen en denominar la GE como un proceso.

Rico (2016) define a la GE como un proceso coordinado que está dirigido a mejorar los procedimientos que existen dentro de las instituciones (educativos, administrativos). Botero (2009) y Giménez (2016) concuerdan en que la GE es el conjunto de acciones, que unen la teoría y la práctica buscando principalmente el perfeccionamiento de las prácticas educativas. Por otro lado, Jahanian \& Motahari (2013) describen la GE como:

Un proceso social que organiza y coordina todos los recursos humanos y materiales mediante la utilización de habilidades científicas, técnicas y artísticas y trata de alcanzar los objetivos de aprendizaje aportando motivaciones y bases de crecimiento y supliendo las necesidades lógicas individualistas y grupales de estudiantes, profesores y empleados. (p. 187)

En cuanto a la GP, Días (2016) la define como "el conjunto coordinado de acciones transversales y mediadoras para el adecuado desarrollo del proyecto de enseñanza escolar" (p.47).

Anteriormente, Antúnez (1996; citado en López, 2017) la había definido de manera más amplia al indicar que la GP "es la agrupación de 
actividades dirigidas a movilizar los recursos (humanos, económicos, inmateriales) hacia el logro de objetivos previamente establecidos, conlleva acciones para analizar los procesos y sus resultados" (p. 207).

En opinión de Islas (2015), la GP se define como la "capacidad de organizar y poner en marcha el proyecto pedagógico de la institución a partir de la definición [qué se debe enseñar y deben aprender los estudiantes]" (p.38).

Por otro lado, Coaquira (2018) implica de manera más puntual el rol del docente, al señalar que la GP es un proceso donde se observa la gestión de las actividades didácticas y 'socio-psico-pedagógicas' llevadas a cabo por la comunidad educativa en miras de la formación integral de los alumnos y, desde la postura de Batista (2007), la GP se asume como el "quehacer coordinado de las acciones y recursos socio-psicopedagógicos y didácticos que realizan los actores sociales de este proceso en el colectivo de año, [buscando direccionar estas acciones] hacia el logro de los objetivos de formación del modelo del profesional (...)" (p.101).

Finalmente, y de acuerdo a las definiciones presentadas, se infiere que la GP se encuentra dentro de un proceso de GE, volviéndose un proceso complejo que contempla a sujetos profesionales de la enseñanza con saberes disciplinares, digitales y didáctico-pedagógico, un saber ser y hacer mediado por la propia vocación en la enseñanza y los valores de la institución educativa. En función de lo anterior, debe saber instrumentar el Modelo Educativo Profesional vigente, buscando cumplir con necesidades de actuación profesional en el saber convivir y crecer en sociedad, buscando mejorar la calidad de vida de todos los ciudadanos. Además, para el funcionamiento integral de la GP debe existir una implicación de todos los actores sociales responsables de las actividades y tareas de aprendizaje que guíen y acompañen de manera eficiente y eficaz, hacia una enseñanza-aprendizaje colaborativa, reflexiva, innovadora, creativa y autónoma.

\section{Marco teórico-contextual}

De acuerdo con Rico (2016) la gestión permite transformar los entornos educativos, siendo conveniente analizar su aplicación en las instituciones. Es dentro de ese entorno que suele denominarse gestión educativa, acentuando el nivel de gestión realizado por los docentes.

Para Camacho et al. (2019), "la gestión llevada a cabo desde una visión docente, ayuda a proveer de realidades académicas complejas obligando a asumir compromisos educativos [acorde a las necesidades que se van presentado en el día a día de la enseñanza-aprendizaje]..." (p.1). Por lo que la gestión educativa concebida desde la complejidad de las distintas realidades que se viven y abordada desde una responsabilidad conjunta, se vuelve crucial para dar respuesta tanto a las demandas laborales como sociales. 
Por otro lado, se sabe que aun cuando las teorías de la administración han servido como precedente para la administración escolar, se considera que además de administrar las escuelas, es preciso gestionarlas.

Algunos autores aseveran que la gestión y la administración muestran diferencias de aplicación; es decir, la gestión orquesta los procesos 'teóricos y prácticos' buscando el continuo de la calidad sobre los mismos y la administración puntualiza en 'el manejo y uso de los recursos' (Chacón, 2014; García, Juárez \& Salgado, 2018). Esta aplicación de la gestión en el ámbito educativo es denominada 'gestión educativa'.

En sus orígenes, Casassus (2000) menciona que, desde la década de los setenta, Estados Unidos fue uno de los países pioneros en aplicar este concepto, sucedido por Inglaterra y, finalmente, adoptado en América Latina en los ochenta.

Autores como Botero (2009), López (2017), Rico (2016) y Romero (2018), coinciden en definir a la gestión educativa como un proceso para el logro de los objetivos institucionales. Botero (2009), además, indica que la gestión educativa en nivel superior debe llevar hacia la autonomía, democracia, formación de calidad, formación integral y educación virtual.

López (2017), por su lado, argumenta que la gestión educativa brinda soporte a los proyectos escolares, conserva la libertad institucional, da cumplimiento a las necesidades políticas y sociales mediante la retroalimentación y enriquecimiento de los procesos de enseñanzaaprendizaje.

Barrios (2018), por otro lado, afirma que una gestión educativa de calidad permite innovar en los procesos de enseñanza y aprendizaje cambiando las estructuras tradicionales por métodos innovadores, para enfrentar los retos del entorno educativo.

En México, la Secretaría de Educación Pública (SEP) (2010) expone que la gestión, en el contexto educativo y en base a sus funciones y niveles de concreción, se clasifica en tres tipos: Gestión Institucional (estructura), Gestión Escolar (comunidad educativa) y Gestión Pedagógica (aula). De esta manera, los tres tipos de gestión convergen o integran el modelo de la Gestión Educativa.

Es decir, estas tendencias, en teoría, han sido consideradas en las políticas educativas del país, las cuáles buscan mejorar la 'gestión en la educación' (Farfán \& Reyes, 2017), al procurar una educación equitativa y de calidad interesada en impartir aprendizajes trascendentes para todos los estudiantes sin distinción, buscando innovar en los procesos de enseñanzaaprendizaje.

En el desarrollo de la gestión educativa las instituciones han puesto mayor interés en la parte administrativa, descuidando la gestión pedagógica (Ibarra, 2016). 
Las investigaciones sobre la gestión GP en Latinoamérica suelen ser de corte cualitativo. En lo que respecta a México, y en específico en el Sur del país, no existen suficientes investigaciones respecto a la GP en nivel superior; sin embargo, hay importantes aportaciones que mencionan las tendencias a tomar en cuenta en este siglo XXI. Dentro de esta vertiente existen propuestas y trabajos que señalan la importancia de renovar la educación superior en México y de establecer políticas públicas que impulsen el cambio institucional (ANUIES, 2018; Botero, 2009; Camacho, et al., 2019; Cerdas, Marín, \& Vargas, 2008; Chacón, 2014; Cueva, 2020; Días, 2016; Farfán \& Reyes, 2017; García, Juárez \& Salgado, 2018; Ibarra, 2016; Jurado, et al., 2011; López, 2017; Penalva, Hernández \& Guerrero, 2013; Rico, 2016; Rodríguez, 2005; Romero, 2018; Ruiz \& León, 2012; SEP, 2010; entre otros).

En la opinión de Jurado, et al. (2011), la implementación de la gestión en la pedagogía es importante porque permite cohesionar los objetivos con los intereses de la institución, además de que fomenta la intervención de los diversos actores educativos.

Para Penalva, Hernández \& Guerrero (2013), las acciones realizadas para alcanzar la eficacia en las instituciones están conectadas con la práctica de los docentes, puesto que son los encargados de desarrollar la gestión pedagógica.

Días (2016) expone que la GP es investigada de forma somera y que carece de sustentos teóricos-conceptuales, lo anterior dificulta concebir una definición; no obstante, la SEP (2010) la describe como una disciplina reciente que engloba 'teorías, políticas y la praxis educativa', además de la aplicación de planes de estudios y prácticas pedagógicas.

Por su parte, López (2017) reconoce que la GP es un proceso que permite organizar las actividades de los docentes priorizando la 'formación integral' de los estudiantes, para responder a las necesidades del entorno.

Para Ibarra (2016) y Rodríguez (2005), la GP precisa de reflexión, colaboración y gestión para generar progresos en la educación.

Cerdas, Marín \& Vargas (2008) mencionan y explican las etapas para el desarrollo de modelos de GP y que orientan hacia la innovación en los centros educativos, siendo estas: (1) Diagnóstico: se analiza el contexto interno y externo, identificando el problema principal; (2) Planeación: se determina las etapas y/o estrategias a seguir; (3) Implementación: se ejecuta lo planeado; y los puntos (4) y (5) comprenden la verificación y los ajustes.

Comprender la GP permite entender la importancia de su aplicación en las IES y mejorar el proceso educativo, el cuál es interdependiente a la calidad educativa (Barrios, 2018; López, 2017), siendo este último concepto el bien más anhelado por las universidades. 


\section{Análisis de Campo}

El trabajo de campo se llevó a cabo dentro de un proyecto académico denominado "Desarrollo de Competencias Docentes en la Investigación Acción Colaborativa", cuyo tiempo de desarrollo fue desde enero del 2018 hasta enero de 2020. Los docentes partícipes realizaron innovaciones en los procesos de enseñanza-aprendizaje, con la premisa de que el trabajo interdisciplinar permite alcanzar la anhelada calidad educativa.

Como antecedente, antes del 2010, para muchos docentes de la universidad, la enseñanza se traducía en transmitir sus conocimientos, estilo comúnmente denominado tradicional. Sin embargo, a pesar de las estrategias incluidas en el Modelo Educativo de la universidad, que entró en vigor desde el 2005 y las consecuentes capacitaciones en competencias docentes, a la fecha, este modelo apenas se ha sabido instrumentar. Situación que ha dejado en desventaja educativa a la institución y que abona a un problema mayor, el constante cambio y afectación contextual que vive la sociedad actual, comprobando lo expuesto por el Comité de Planeación para el Desarrollo del Estado de Tabasco (COPLADET, 2019): "la educación evoluciona tan rápido que los instrumentos y métodos que se utilizan por un tiempo corren el riesgo de quedar rezagados" (p.4).

En cuanto al enfoque por competencias, este busca potenciar las capacidades, la innovación y la creatividad de los estudiantes mediante el desarrollo de competencias (habilidades, destrezas, aptitudes y actitudes) que les permitan enfrentar diversas situaciones en el contexto actual, implica una visión holística en la educación y el docente lleva un rol protagónico, puesto que es el encargado de formar en competencias a los estudiantes (García, 2011).

Se afirma la necesidad de transición de la labor docente de un modelo tradicional, caracterizado por la transmisión del conocimiento, a un modelo enfocado en el aprendizaje (Sierra, Sevilla \& Martín, 2019). La Asociación Nacional de Universidades e Instituciones de Educación Superior (ANUIES) (2018), converge en la importancia de trabajar bajo nuevos enfoques educativos para facilitar el aprendizaje en la vida, los cuales permitan a los individuos desarrollar competencias e interactuar con la compleja realidad.

\section{La reflexión en la gestión pedagógica}

A pesar que para muchos docentes ha sido difícil la transición a este nuevo enfoque, el grupo de docentes participantes en el proyecto manifestó pleno interés en trascender su enseñanza; por lo que se sumergieron en una etapa de reflexión sobre su labor bajo este nuevo enfoque del modelo educativo ya que, como menciona Cueva (2020), el docente que no problematiza ni reflexiona sobre su praxis, no percibe la necesidad de modificar su actuación. De esta manera, el grupo de docentes participantes 
indagó, comprendió, reflexionó y acordó trabajar una planeación didáctica utilizando la metodología del Aprendizaje Orientado a Proyectos o Basado en Proyectos (AOP/ABP).

\section{Gestión pedagógica en el proceso profesionalizante}

Cerdas, et al. (2008) y López (2017) coinciden al señalar los pasos siguientes como esenciales para una buena gestión pedagógica: diagnóstico, planeación, ejecución, seguimiento y evaluación en el aprendizaje. El grupo de investigadores en estudio coincidieron en los pasos anteriores, pero aplicados no sólo al aprendizaje de los estudiantes, sino al desarrollo de las competencias docentes. Buscando llevar al docente, de manera voluntaria, hacia un proceso profesionalizante de la enseñanza, más proactivo e innovador. Este proceso se estableció de la siguiente manera:

1. Reclutamiento Voluntario: una de las finalidades perseguidas por este proyecto es fomentar el trabajo cooperativo/colaborativo entre docentes, por lo que se realizó la invitación de manera personalizada a los docentes para participar en este proyecto.

2. Diagnóstico: en esta etapa los docentes expusieron y reconocieron, que se sigue calificando a los estudiantes por el nivel de memorización. La opción didáctica elegida por el docente en el aprendizaje áulico, son las exposiciones por equipo; también se reconoció la falta de cumplimiento y seguimiento de los programas académicos de la universidad y la no fomentación del trabajo cooperativo/colaborativo entre los propios colegas. Asimismo, se pudo diagnosticar que en la planta docente se encuentran profesionales sin ninguna formación pedagógica, puesto que para que un profesional sea contratado por la universidad basta con contar con una experiencia de por lo menos cuatro años en docencia o tener el grado de doctor.

Conjuntamente, se identificó que el área de Coordinación de Docencia (encargada de la formación profesional y pedagógica) tiene una vinculación relativamente baja con los docentes, excluyendo uno de sus objetivos: la coordinación y gestión de actividades académicas que vinculen a los docentes en un trabajo de equipo para lograr una educación de calidad.

3. Inducción: esta fase fue realizada antes del inicio del ciclo escolar, se capacitó a los docentes con conocimientos teóricos-prácticos sobre el entorno mixto y métodos de aprendizaje. En cada taller ofertado, antes de iniciar los ciclos académicos, se buscó enlazar, reflexionar y retroalimentar los conocimientos adquiridos, con la finalidad de reforzar y aprehender estos al nuevo rol docente.

4. Planeación didáctica: se realizaron reuniones donde fueron planeadas de manera conjunta las estrategias de aprendizaje, para impartir los 
contenidos curriculares. Se diseñaron actividades que reforzaron las partes teóricas de las asignaturas a su cargo, fomentando el desarrollo del pensamiento crítico de sus alumnos y el trabajo en equipo. Como se decidió trabajar con la metodología del aprendizaje orientado a proyectos o basado en proyectos (ABP/AOP), se establecieron las pautas y consideraciones respecto a estos proyectos.

También se buscó estimular la creatividad y el uso de las tecnologías en los estudiantes mediante la creación del Portafolio de Evidencia Virtual (PEV) en la plataforma Wix, en donde los docentes crearon también sus propias páginas, denominada "Blog de Actuación Educativa", agregando materiales de consulta e interés para reforzar los contenidos enseñados en el salón de clases, buscando generar un ambiente de aprendizaje mixto.

Respecto a la decisión de incluir un portafolio de evidencias, Loera, García y Cázares (2011) mencionan que el portafolio "es un instrumento que facilita la mejora de la enseñanza y el aprendizaje... centrado en la evidencia del aprendizaje y orientado a la reflexión sobre lo aprendido y el proceso de aprendizaje mismo." (p. 65).

5. Ejecución: todo lo acordado en la planeación didáctica entre los docentes fue puesto en marcha en los ciclos escolares desde agosto de 2017 hasta enero de 2020.

6. Seguimiento e intervención durante el ciclo académico: durante el ciclo escolar se realizaron reuniones periódicas donde los docentes participaron de manera activa describiendo su sentir y compartiendo aspectos relevantes que sucedieron en el aula como: las oportunidades observadas y las aptitudes que percibieron en sus alumnos. De acuerdo con Cueva (2020) el trabajo colaborativo de reflexión entre colegas es fundamental en el proceso de la gestión pedagógica, puesto que permite reconocer fortalezas y debilidades además de aprender al escuchar las experiencias de otros docentes.

7. Socialización de la enseñanza-aprendizaje por competencias: al final del ciclo escolar, fueron seleccionados los proyectos destacados de cada salón los cuáles se presentaron en una exposición académicoempresarial, donde se invitó a empresarios, directivos, docentes y alumnos a conocer los resultados del proceso enseñanza-aprendizaje de estos estudiantes.

8. Retroalimentación conjunta de la actuación docente: al término del semestre se realizó la actividad de retroalimentación entre docentes,

${ }^{2}$ Ver ejemplo de uno de los Blog de Actuación Educativa en https://mcj308.wixsite.com/ciclos 
para analizar de manera conjunta su trabajo durante el ciclo y los resultados obtenidos.

Este proceso ha generado resultados de avance con paso firme $y$ seguro, el cual es reflejado con los docentes participantes involucrados en el proyecto y con los grupos de alumnos donde se aplicó la planeación por competencias. En los momentos de reflexión ${ }^{3}$, tanto los docentes como los estudiantes, manifestaron que al inicio se le dificultó trabajar de esta manera pero, posteriormente, se sintieron motivados al corroborar las competencias y habilidades profesionales adquiridas durante el ciclo escolar.

Es así como los resultados de este proyecto permitieron observar las fortalezas y las debilidades de desarrollo de la gestión pedagógica dentro de un proceso profesionalizante, ya que se describe y se reflexiona sobre el mismo.

\section{Conclusión reflexiva}

El análisis realizado en esta investigación confirma que los procesos de gestión pedagógica permiten que las IES puedan hacer frente a las demandas de la sociedad.

Se reconoce que el proceso de transformación de la educación superior comienza en el aula y la responsabilidad es dirigida, originalmente, hacia los docentes. Por lo que su gestión en el aula es el elemento clave para lograr la formación integral de los estudiantes y la consolidación de una educación de calidad. Sin embargo, es con el trabajo conjunto entre docentes y administrativos como se logra una gestión educativa de calidad, lo que permite el éxito en los procesos (Barrios, 2018; Cárdenas, Farías \& Méndez, 2017, Rico; 2016).

Por otra parte, analizar el proceso profesionalizante desarrollado en el proyecto permitió observar la amalgama de actuaciones y actividades en cada etapa, haciendo posible diagnosticar falencias en el proceso. Como ejemplo de ello, se identificó que se requiere de más apoyo para la socialización de resultados y soporte por parte de la coordinación de docencia y dirección de la división hacia este tipo de proyectos académicos. Como afirman Ruiz \& León (2012) el nivel de motivación en los docentes depende de la organización educativa en donde se desempeñan, si esta presenta una gestión educativa inclusiva, el desempeño docente será destacable mejorando la calidad en la enseñanza.

En México aún existe un camino por recorrer en la GP, como parte de la GE de las IES, desde reconocer su importancia en la labor educativa al

\footnotetext{
${ }^{3}$ Para saber más sobre las etapas reflexivas de los docentes ver ejemplo en https://mcj308.wixsite.com/docenciadacea/copia-de-metodologia-aop y de estudiantes ver ejemplo en: https://mcj308.wixsite.com/ciclos/copia-de-2018-2019.
} 
inculcar la colaboración activa de todos los participantes, hasta dar seguimiento a investigaciones específicas a nivel regional o local que permitan observar, con sustento teórico-práctico, el actuar de la enseñanza-aprendizaje por competencias a nivel nacional, buscando que tomas de decisión política en la educación profesional puedan ser más certeras y congruentes ante la diferencia de realidades que se vive a lo largo de todo el país.

\section{References:}

1. Asociación Nacional de Universidades e Institutos de Enseñanza Superior (ANUIES). (2018). Visión y acción 2030. Propuesta de la ANUIES para renovar la educación superior en México. Diseño y concertación de políticas públicas para impulsar el cambio institucional.

2. Barrios, C.E. (2018). Gestión educativa basada en un sistema de calidad en instituciones de educación universitaria. Revista Docencia Universitaria, 19 (1), 53-73.

3. Batista, T. (2007). La gestión pedagógica en el año académico desde un modelo integrador del currículo y la labor educativa. Revista Institucional Tecnológica del Chocó. D.L.C., 6, 99-104

4. Behar, D. (2008). Metodología de la investigación. Ediciones Shalom.

5. Botero, C. (2009). Cinco tendencias de la gestión educativa. Revista Iberoamericana de educación, 49 (2), 17-31.

6. Camacho, M., López, J. C., Camacho, D., y Reyes, R. (2019). Gestión Educativa en la Profesionalización de la Enseñanza y el Aprendizaje por Competencias: Caso "DACEA-CUC de la UJAT". En Difusión de experiencias y resultados de investigación a nivel superior: Chiapas 2019 [Tomo 9], 1373-1378. Libro Digital con ISBN 978-1-96998251-3

7. Cárdenas, C., Farías, G. \& Méndez, G. (2017). ¿Existe relación entre la gestión administrativa y la innovación educativa? Un estudio de caso en educación superior. Revista Iberoamericana sobre Calidad, Eficacia y Cambio en Educación, 15 (1), 19-35.

8. Casassus, J. (2000). Problemas de la gestión educativa en América Latina (la tensión entre los paradigmas de tipo A y el tipo B). UNESCO.

9. Cerdas, V., Marín, A. \& Vargas, I. (2008). Modelos de gestión pedagógica, en zona de atención prioritaria: análisis de una experiencia. Revista Educare, 12 (2), 9-29.

10. Chacón, L. (2014). Gestión educativa del siglo XXI: bajo el paradigma emergente de la complejidad. Omnia, 20(2), 150-161. 
11. Coaquira, L. (2018). Gestión pedagógica y estilos de aprendizaje en estudiantes de la institución educativa primaria $N^{\circ} 70173$ glorios 890, Julio-Puno, 2017, (Tesis Maestría) Universidad César Vallejo.

12. Comité de planeación para el desarrollo del estado de Tabasco (COPLADET). (2019). Programa sectorial educación, ciencia, tecnología, juventud y deporte 2019-2024.

13. Cortés, M. \& Iglesias, M. (2004). Generalidades sobre Metodología de la Investigación. Universidad Autónoma del Carmen.

14. Cueva, A. (2020). Trabajo colaborativo docente y su impacto en la gestión pedagógica. Ciencia y Educación, 1 (1), 19-24.

15. Días, P. (2016). Cambio educativo y gestión pedagógica en América Latina: estudio comparativo de los conceptos y procesos de gestión de los liderazgos pedagógicos en escuelas de Educación Básica en México y Brasil, (Tesis doctorado) Universidad Nacional Autónoma de México.

16. Farfán, M. \& Reyes, I. (2017). Gestión educativa estratégica y gestión escolar del proceso de enseñanza-aprendizaje: una aproximación conceptual. Reencuentro. Análisis de Problemas Universitarios, 28 (73), 45-62.

17. García, F., Juárez, S. \& Salgado, L. (2018). Gestión escolar y calidad educativa. Revista Cubana Educación Superior, 37 (2), 206-216.

18. García, J. (2011). Modelo educativo basado en competencias: importancia y necesidad. Revista Electrónica "Actualidades Investigativas en Educación", 11 (3), 1-24.

19. Giménez, A. (2016). El papel de la gestión de centros educativos en un modelo de aprendizaje basado en competencias. Revista Páginas de Educación, 9 (1), 5-15.

20. Hernández Sampieri, R., Fernández Collado, C., \& Baptista Lucio, M. d. (2014). Metodología de la investigación (Sexta ed.). McGraw Hill.

21. Ibarra, L. (2016). Gestión pedagógica. DIGITAL CIENCIA@UAQRO.

22. Islas, M. (2015). Gestión pedagógica basada en el modelo de competencias en el ejercicio profesional de las educadoras en la Zona Escolar 50 Delegación Tlalpan, (Tesis Maestría). Universidad Pedagógica Nacional.

23. Jahanian, R. \& Motahari, M. (2013). Solutions to improve educational management quality. International Journal of Management and Sustainability, 2 (11), 186-192.

24. Jurado, S., Leal, N., Leguizamón, I. \& Sánchez, J. (2011). La gestión educativa y su relación con la pedagogía en la cultura institucional. Revista LOGOS CIENCIA \& TECNOLOGÍA, 2 (2), 220-235. 
25. Loera, A., García, E. \& Cázares, Ó. (2011). Gestión pedagógica centrada en el aprendizaje. Fundación de Empresarios por la Educación Básica de México, A. C.; Heurística Educativa.

26. López, M. (2017). La gestión pedagógica. Apuntes para un estudio necesario. Revista Científica Dominio de las Ciencia, 3, núm. esp., 201-215.

27. Organización de las Naciones Unidas para la Educación, la Ciencia y la Cultura (UNESCO) (2017). La educación al servicio de los pueblos y el planeta: creación de futuros sostenibles para todos. Informe de seguimiento de la educación en el mundo 2016.

28. Otzen, T. \& Manterola, C. (2017). Técnicas de Muestreo sobre una Población a Estudio. Int. J. Morphol, 35 (1), 227-232.

29. Pardo, R., Salazar, M., Díaz, R., Bosco, M., Negrín, M., Del Valle, E., Cerón, A. \& Alcázar, P. (2013) La evaluación en la escuela. Secretaría de Educación Pública.

30. Penalva, A., Hernández, M. \& Guerrero, C. (2013). La gestión eficaz del docente en el aula. Un estudio de caso. Revista Electrónica Interuniversitaria de Formación del Profesorado, 16 (2), 77-91.

31. Rico, A. (2016). La gestión educativa: Hacia la optimización de la formación docente en la educación superior en Colombia. Sophia, 2016, 12 (1), 55-70.

32. Rodríguez, A. (2005). El lugar de la gestión pedagógica en la escuela. Magazín Aula Urbana, (54), 3-13.

33. Romero, G. (2018). Calidad educativa: engranaje entre la gestión del conocimiento, la gestión educativa, la innovación y los ambientes de aprendizaje. Revista de Estudios y Experiencias en Educación, 17 (35), 91-103.

34. Ruiz, E. \& León, G. (2012). Las políticas, las instituciones y la gestión pedagógica: Una mirada a la realidad educativa mexicana. Revista Calidad en la Educación Superior, 3(2), 50-65.

35. Secretaría de Educación Pública (SEP) (2010). Programa Escuelas de Calidad. Gobierno Federal.

36. Sierra, T., Sevilla, D. y Martín, M. (2019). Profesor universitario, ser en resiliencia: una mirada a su labor en el contexto educativo actual. Diálogos sobre educación. Temas actuales en investigación educativa, 10 (19). 